\title{
Chemical abundances of giant stars in the Crater stellar system $^{\star, \star \star}$
}

\author{
P. Bonifacio ${ }^{1}$, E. Caffau ${ }^{1, \star \star \star}$, S. Zaggia ${ }^{2}$, P. François ${ }^{1,3}$, L. Sbordone ${ }^{4,5}$, S. M. Andrievsky ${ }^{6,1}$, and S. A. Korotin ${ }^{6}$ \\ ${ }^{1}$ GEPI, Observatoire de Paris, PSL Research University, CNRS, Univ. Paris Diderot, Sorbonne Paris Cité, Place Jules Janssen, \\ 92195 Meudon, France \\ e-mail: Piercarlo.Bonifacio@obspm.fr \\ ${ }^{2}$ Istituto Nazionale di Astrofisica, Osservatorio Astronomico di Padova, Vicolo dell'Osservatorio 5, 35122 Padova, Italy \\ 3 UPJV, Université de Picardie Jules Verne, 33 rue St Leu, 80080 Amiens, France \\ ${ }^{4}$ Millennium Institute of Astrophysics, Casilla 306, Santiego 22, Chile \\ 5 Pontificia Universidad Católica de Chile Vicuña MacKenna 4860, Macul, Santiago, Chile \\ 6 Department of Astronomy and Astronomical Observatory, Odessa National University, and Isaac Newton Institute of Chile Odessa \\ branch, Shevchenko Park, 65014 Odessa, Ukraine
}

Received 20 April 2015 / Accepted 9 June 2015

\section{ABSTRACT}

\begin{abstract}
Aims. We obtained spectra for two giants of Crater (Crater J113613-105227 and Crater J113615-105244) using X-Shooter at the VLT, with the purpose of determining their radial velocities and metallicities.

Methods. Radial velocities were determined by cross-correlating the spectra with that of a standard star. The spectra were analysed with the MyGIsFOS code using a grid of synthetic spectra computed from one-dimensional, local thermodynamic equilibrium (LTE) model atmospheres. Effective temperature and surface gravity were derived from photometry measured from images obtained by the Dark Energy Survey.

Results. The radial velocities are $144.3 \pm 4.0 \mathrm{~km} \mathrm{~s}^{-1}$ for Crater J113613-105227 and and $134.1 \pm 4.0 \mathrm{~km} \mathrm{~s}^{-1}$ for Crater J113615-105244. The metallicities are $[\mathrm{Fe} / \mathrm{H}]=-1.73$ and $[\mathrm{Fe} / \mathrm{H}]=-1.67$, respectively. In addition to the iron abundance, we were able to determine abundances for nine elements: $\mathrm{Na}, \mathrm{Mg}, \mathrm{Ca}, \mathrm{Ti}, \mathrm{V}, \mathrm{Cr}, \mathrm{Mn}, \mathrm{Ni}$, and $\mathrm{Ba}$. For $\mathrm{Na}$ and $\mathrm{Ba}$ we took into account deviations from LTE because the corrections are significant. The abundance ratios are similar in the two stars and resemble those of Galactic stars of the same metallicity. In the deep photometric images we detected several stars that lie to the blue of the turn-off.

Conclusions. The radial velocities imply that both stars are members of the Crater stellar system. The difference in velocity between the two taken at face value implies a velocity dispersion $>3.7 \mathrm{~km} \mathrm{~s}^{-1}$ at a $95 \%$ confidence level. Our spectroscopic metallicities agree excellently well with those determined by previous investigations using photometry. Our deep photometry and the spectroscopic metallicity imply an age of $7 \mathrm{Gyr}$ for the main population of the system. The stars to the blue of the turn-off can be interpreted as a younger population that is of the same metallicity and an age of 2.2 Gyr. Finally, spatial and kinematical parameters support the idea that this system is associated with the galaxies Leo IV and Leo V. All the observations favour the interpretation of Crater as a dwarf galaxy.
\end{abstract}

Key words. stars: abundances - stars: Population II - globular clusters: general - galaxies: abundances - Local Group

\section{Introduction}

The compact stellar system Crater has been independently discovered by Belokurov et al. (2014) from the ATLAS ESO VST survey $^{1}$ (Shanks et al. 2013) supplemented with deep imaging with the WHT 4m telescope, and by Laevens et al. (2014) from the Pan-STARRS1 Survey ${ }^{2}$ (Chambers \& PS1 Science Consortium 2015), supplemented by deep photometry with ESO/MPG $2.2 \mathrm{~m}$ telescope. Laevens et al. (2014) referred to the system as PSO J174.0675-10.8774, in the present paper we adopt the naming proposed by Belokurov et al. (2014), which is more in line with the current convention of naming new dwarf

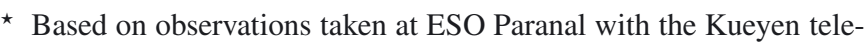
scope, programme 094.D-0547.

$\star \star$ Tables 3-4, Figs. 4-5, and Appendices are available in electronic form at http: //www . aanda. org

$\star \star \star$ MERAC fellow.

1 http://astro.dur.ac.uk/Cosmology/vstatlas/

2 http://pan-starrs.ifa.hawaii.edu/public/
}

galaxies from the constellations in which they reside: Crater. The two investigations substantially agree with respect to the distance of the system: between $145 \mathrm{kpc}$ and $170 \mathrm{kpc}$ for Belokurov et al. (2014) and $145 \mathrm{kpc} \pm 17 \mathrm{kpc}$ according to Laevens et al. (2014). They also agree on the estimate of the metallicity of the system: $<-1.8$ according to Belokurov et al. (2014), -1.9 according to Laevens et al. (2014). However, the two investigations depart significantly as to the nature of the stellar system. Belokurov et al. (2014) interpreted it as a dwarf galaxy satellite of the Milky Way, although the hypothesis that it is a peculiar globular cluster (GC) is also discussed. This interpretation is supported by the existence of a few bright blue stars present within in the stellar system, which can be interpreted as young blue-loop giants, resulting from a recent star formation episode. In addition, in support of the dwarf galaxy interpretation, Belokurov et al. (2014) pointed out that the horizontal branch (HB) of the system is quite red and not very extended: such a morphology of the HB is not compatible with low metallicity. Low-metallicity GCs show HBs that are very 


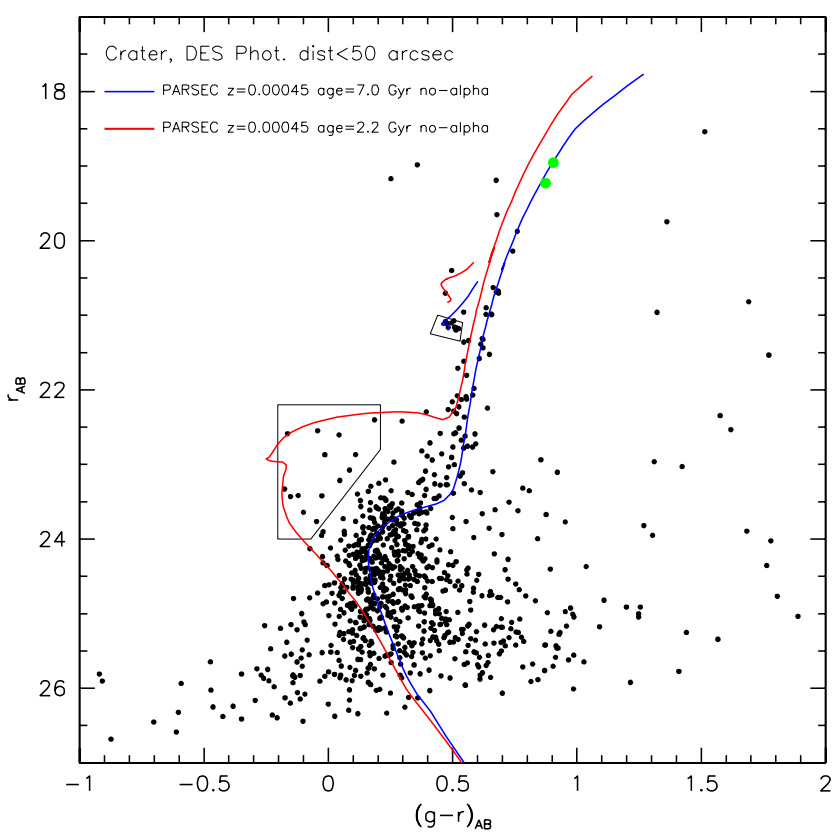

Fig. 1. Colour-magnitude diagram in $g$ and $r$ bands of the Crater stars within 50.'0 from the centre, based on the DES public images. The two target stars are identified as large green dots. Overplotted are shown two PARSEC isochrones for a $7 \mathrm{Gyr}$ and a $2.2 \mathrm{Gyr}$ old stellar population. A reddening correction of $E(B-V)=0.023$, as deduced from the maps of Schlegel et al. (1998), has been applied to the isochrones.

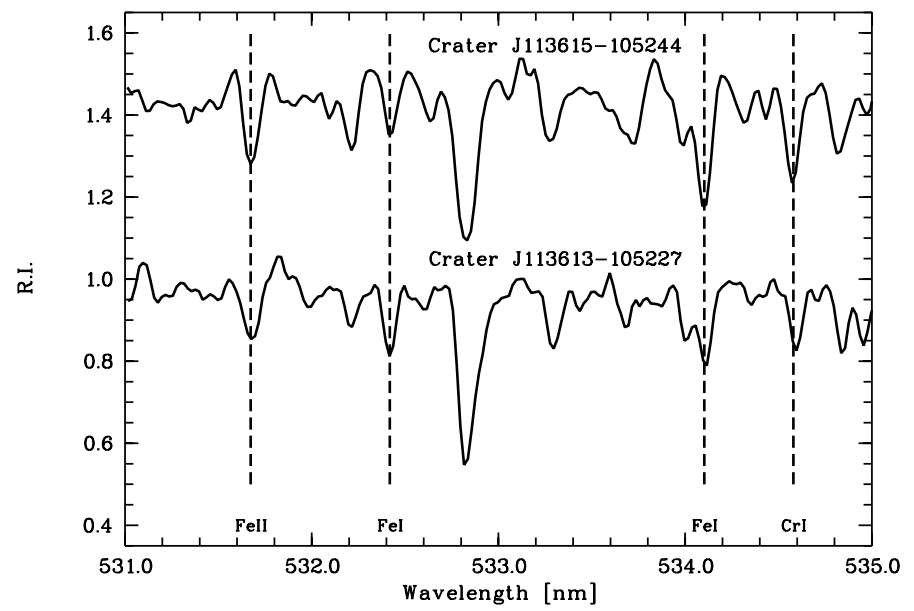

Fig. 2. Portion of the X-Shooter normalised spectra of the two stars, R.I. is the residual intensity. The spectrum of Crater J113615-105244 has been displaced vertically by 0.5 for display purposes. Only lines that have been used in the abundance analysis are identified.

extended to the blue. Laevens et al. (2014) instead interpreted the system as a distant GC, for which they derived a precise distance, metallicity, and age ( $8 \mathrm{Gyr}$ ). If this interpretation is correct, then the bright blue stars observed in the system are either blue stragglers or non-members. It should be stressed that no other such "young" metal-poor GC has been observed to date. Such a system could have been formed in a currently disrupted satellite galaxy and now been accreted to the Milky Way halo.

In this Letter we present the result of spectroscopic observations of two of the brightest giants of Crater. Our main motivation was to determine radial velocities and metallicities for the two stars.

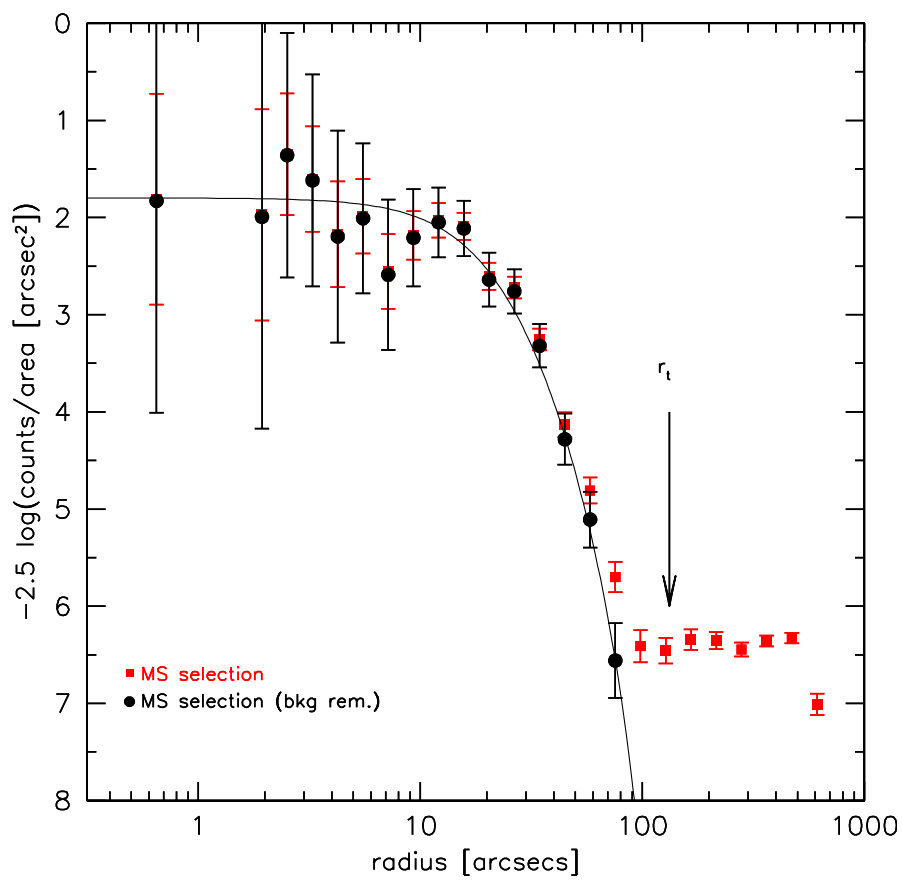

Fig. 3. Radial profile of Crater. The red squares are the raw counts of Crater stars selected along the stellar sequence; the filled circles represent the counts corrected for foreground contamination using the colour-magnitude diagram. The solid line is the best-fitting King model (King 1966), and the position of the tidal radius is indicated by a downward arrow.

\section{Target selection, observations, and analysis}

In the two photometries presented by Belokurov et al. (2014) and Laevens et al. (2014), two luminous red giant stars are visible at $g \approx 20$ at $\simeq 2 \mathrm{mag}$ over the HB. From our previous experience with X-Shooter and the Exposure Time calculator, we estimated that the stars were bright enough to obtain a spectrum suitable for measuring radial velocities with $\mathrm{X}$-Shooter and determine abundances in about four hours of exposure for each star. We retrieved $g, r$ public images from the ESO archive and catalogues of Data Release 1 of VST ATLAS and used them to identify the stars and provide the coordinates used for the observation. Since magnitudes in the catalogue were obtained with aperture photometry, we ran DAOPHOT/ALLSTAR on the released images to extract PSF photometry to improve the coordinates and photometry of the targets, which are already at the detection limit. The final photometry was corrected for illumination using the ATLAS DR1 catalogue.

We subsequently retrieved public $g, r$ deep images observed in the course of the Dark Energy Survey (DES; Abbott et al. 2005; Diehl et al. 2014) from the NOAO Science Archive ${ }^{3}$. We used two images, obtained from the stacking of several short exposures, for each band for a total time of $6240 \mathrm{~s}(3720+2520)$. The seeing of the images is quite good $\simeq 0.8 \div 0.9$ arcsec. We extracted PSF photometry with DAOPHOT/ALLSTAR and used the multiple exposures to obtain a deeper photometry with ALLFRAME. The final $g, r$ catalogue was calibrated with the ATLAS DR1 photometry, which is tied to the APASS system (Henden \& Munari 2014; Munari et al. 2014). Magnitudes are AB magnitudes and should be on the DECAM system. The DES adopted photometry was used to create the $g, r$ colour-magnitude diagram of Crater as shown in Fig. 1, where the two observed

3 http://portal-nvo.noao.edu/ 
Table 1. Observing and measured parameters of our programme stars.

\begin{tabular}{lrr}
\hline \hline Parameter & J113613-105227 & J113615-105244 \\
\hline RA (J2000.0) & $11: 36: 13.90$ & $11: 36: 15.94$ \\
Dec (J2000.0) & $-10: 52: 27.40$ & $-10: 52: 44.10$ \\
$r[\mathrm{mag}]$ & 18.954 & 19.230 \\
$(g-r)[\mathrm{mag}]$ & 0.905 & 0.874 \\
$E(B-V)[\mathrm{mag}]$ & 0.023 & 0.023 \\
\hline$v_{\text {rad }}\left[\mathrm{km} \mathrm{s}^{-1}\right]$ & $144.3 \pm 4$. & $134.1 \pm 4$. \\
$T_{\text {eff }}[\mathrm{K}]$ & 4575 & 4643 \\
$\log g[\mathrm{cgs}]$ & 1.2 & 1.4 \\
$\xi[\mathrm{kms}]$ & 2.0 & 2.0 \\
{$[\mathrm{Fe} / \mathrm{H}][\mathrm{dex}]$} & -1.73 & -1.67 \\
{$[\alpha / \mathrm{Fe}][\mathrm{dex}]$} & 0.34 & 0.41 \\
\hline
\end{tabular}

Table 2. Results of the fit of a King model to the MS stars of Crater.

\begin{tabular}{lrr}
\hline \hline Quantity & Best fit & Error \\
\hline Core radius & $26, .5$ & $4{ }^{\prime \prime} 3$ \\
Tidal rdius & 132 '.5 & $15^{\prime \prime} 2$ \\
Concentration & 0.70 & 0.12 \\
\hline
\end{tabular}

stars are highlighted in green. Coordinates and photometry for our stars are given in Table 1 . The two stars are quite centrally located in Crater. Our observing programme was to observe each star with X-Shooter (Vernet et al. 2011) with the Integral Field Unit, which re-images an input field of $4^{\prime \prime} \times 1.8^{\prime \prime}$ onto a pseudo slit of $12^{\prime \prime} \times 0 .{ }^{\prime} 6$ (Guinouard et al. 2006). Four observing blocks (OBs) of one hour each should have provided $12136 \mathrm{~s}$ of integration on each star as well as a resolving power of $R=7900$ in the UVB arm and 12600 in the VIS arm. Four OBS on Crater J113613-105227 were executed, but only two OBs on Crater J113615-105244. Although with a lower signalto-noise than we had expected, the latter spectra turned out to be of sufficient quality for our analysis. The spectra were reduced as described in Caffau et al. (2011). The signal-to-noise ratio of the coadded spectra at $530 \mathrm{~nm}$ was about 60 for Crater J113613105227 and about 40 for Crater J113615-105244. A portion of the spectra is shown in Fig. 2.

\subsection{Photometry}

We used the DES deep photometry to measure the size of Crater. From the colour-magnitude diagram we selected Crater stars brighter than $r=25.0$ (i.e. $\simeq 1$ mag below the turn-off, TO) along the old stellar sequence taking into account the local errors in colour, and we used this sample to determine the centre of Crater. We excluded the blue stars above the TO and counted the number of stars per unit area in radial annuli as a function of distance from the centre. The results of these counts are shown in Fig. 3. We fitted a King model (King 1966) to these number counts, and the results of our best fit are provided in Table 2. These compare well to what was determined by Laevens et al. (2014) assuming a King model, although we point out that we derived a smaller core-radius and a larger (almost a factor of 2) tidal radius. The reason is probably that DES photometry is deeper than the WFI photometry of Laevens et al. (2014). The colour-magnitude diagram shown in Fig. 1 for the stars $\leq 50$ ' 0 or $\leq 1.9$ core radii from the centre confirms that the dominant population of Crater is of intermediate age. The PARSEC isochrones of the Padova Group (Bressan et al. 2012) in the DECAM AB system for a metallicity of $[\mathrm{Fe} / \mathrm{H}]=-1.5\left(\mathrm{Z}=4.5 \times 10^{-4}\right)$, and reddened for the extinction expected for Crater from the Schlegel et al. (1998) maps, are in favour of a younger age of $\simeq 7$ Gyr compared to the analysis of Belokurov et al. (2014). We do not see a very young population of $\simeq 400 \mathrm{Myr}$, but the good seeing and cleaner photometry allow us to identify a population of objects $\approx 1.5$ mag above the turn-off region. We visually inspected these objects to verify that they are not background compact galaxies or image artefacts, and they all appear to be stellar objects. The younger population is compatible with an age of $\sim 2.2$ Gyr and the metallicity is compatible with the one of the main population. A population of blue stragglers with a mass of $\simeq 1.4 M_{\odot}$. might account for some of the younger potential MS population, but the almost horizontal track of points heading toward the sub-giant branch does not follow an expected blue-straggler locus. With our analysis we measured a distance modulus of $(M-m)_{0}=20.91 \pm 0.1 \mathrm{mag}$ or a distance of $152 \mathrm{kpc}$, confirming the distance found by the previous authors.

\subsection{Radial velocity}

We measured the radial velocities given in Table 1 by crosscorrelating each individual exposure in the UVB arm against the X-Shooter spectrum of HD 165195, taken from the X-Shooter spectral library (Chen et al. 2014). This star has atmospheric parameters quite close to our programme stars. We then applied the barycentric correction to the radial velocities and added a zeropoint correction. The zero-point correction was derived for each exposure from the [OI] $577.7 \mathrm{~nm}$ atmospheric emission line. The zero-point corrections are probably due to a combination of flexures in the spectrograph, variation in temperature, and pressure in the surroundings with respect to when the calibration $\mathrm{Th}-\mathrm{Ar}$ was taken, as well as centring of the star on the IFU. The use of an emission line is not optimal for this purpose, since the illumination of the slit is different for the sky emission and for the star, but we do not have strong enough telluric absorprion lines to use in the UVB arm. Interestingly, Schönebeck et al. (2014) have performed several measurements of the [OI] $577.7 \mathrm{~nm}$ line at different positions on the slit on X-Shooter slit spectra, and the dispersion of their measurements is of only $0.6 \mathrm{~km} \mathrm{~s}^{-1}$. We cannot make use of the VIS spectra for the radial velocities for two reasons: since the stars are metal-poor, there are few lines in the VIS range, and the observations were made by tracking at $470 \mathrm{~nm}$, thus the UVB spectrum is expected to remain well centred, while the VIS spectrum probably shows larger deviations during the exposure ${ }^{4}$.

Then we computed a weighted average of the radial velocities, with weights that are proportional to the number of counts in the spectrum in the region $490-550 \mathrm{~nm}$. The use of a straight average changes the mean by less than $0.1 \mathrm{~km} \mathrm{~s}^{-1}$.

Measuring the radial velocities on the indivudal spectra has the additional advantage that the dispersion in the measurements provides an estimate of the error. For Crater J113613-105227, this is $4 \mathrm{~km} \mathrm{~s}^{-1}$, while it is $0.4 \mathrm{~km} \mathrm{~s}^{-1}$ for Crater J113615105244. We take the former as our error estimate, since the spectra were taken on three different nights and probe different instrument flexures, ambient temperature, and star centring, while the two spectra of CraterJ113615-105244 were taken on the same night, one after the other. The latter do provide an estimate of the statistical error in our radial velocity measurements. Our error estimate is in line with the quoted accuracy of the calibration of the X-Shooter UVB and VIS

4 See the X-Shooter manual-page $27 \mathrm{https://www.eso.org/}$ sci/facilities/paranal/instruments/xshooter/doc/ VLT-MAN-ESO-14650-4942_P96.pdf 
arms (Goldoni 2011, $2 \mathrm{~km} \mathrm{~s}^{-1}$ ). The difference in radial velocity, $10.2 \mathrm{~km} \mathrm{~s}^{-1}$, allows us to confirm that the two stars belong to the same stellar system. This was expected because the stars are near the centre of Crater, as can be appreciated in Fig. 4. The average radial velocity of Crater is $139.2 \pm 4 . \mathrm{km} \mathrm{s}^{-1}$. The radial velocity in the Galactic standard of rest is $v(\mathrm{GSR})=-5.7 \pm 4 . \mathrm{km} \mathrm{s}^{-1}$ using the Dehnen \& Binney (1998) correction for the solar motion and a correction for the rotation of the Galaxy of $220.0 \mathrm{~km} \mathrm{~s}^{-1}$.

\subsection{Abundance analysis}

To analyse the spectra, we used our code MyGIsFOS (Sbordone et al. 2014) and a grid of synthetic spectra appropriate for metalpoor giants, computed from our grid of ATLAS 12 models (Sbordone et al., in prep.; Kurucz 2005; Castelli 2005) covering the metallicity range -3.0 to -1.0 . The spectra were computed assuming local thermodynamic equilibrium (LTE). For both stars we adopted the atmospheric parameters as read from the $7 \mathrm{Gyr}$ isochrone that is shown in Fig. 1. The adopted surface gravites are also supported by the iron ionisation equilibria, even though we have only two Fe II lines in Crater J113613105227 and one in Crater J113615-105244. MyGIsFOS is also capable of determining the temperature from the Fe I excitation equilibrium. Since in metal-poor giants the Fe I lines can deviate significantly from LTE (see e.g. Mashonkina et al. 2011, and references therein), we decided that the isochrones and the colourmagnitude diagram provide a more robust estimate of the effective temperature. It is not possible to measure lines on the linear part of the curve-of-growth at the resolution of X-Shooter, thus one has no information on the microturbulent velocity. This parameter, which has to be introduced when using one-dimensional static model atmospheres as done here, depends on the atmospheric parameters. We decided to adopt $2.0 \mathrm{~km} \mathrm{~s}^{-1}$ for the microturbulent velocity because this was measured by Cayrel et al. (2004) for HD 122563. This star has a $T_{\text {eff }}$ and $\log g$ similar to our stars, but a lower metallicity. Monaco et al. (2005) derived a calibration of the microturbulent velocity as a function of $T_{\text {eff }}$ and $\log g$ for metallicities in the range -0.5 to -1.0 . Their calibration implies microturbulent velocities $\approx 1.8 \mathrm{~km} \mathrm{~s}^{-1}$.

For $\mathrm{Na}$ and $\mathrm{Ba}$, which are known to be strongly affected by deviations from LTE, we derived the abundances from fitting line profiles with theoretical profiles computed in NLTE with the code MULTI (Carlsson 1986) as modified by Korotin et al. (1999) and Korotin \& Mishenina (1999). For Na we relied on the NaI D doublet, the model atom used is the same as in Andrievsky et al. (2007). To derive the Ba abundances, we used the the $614.15 \mathrm{~nm}$ Ba II line for both stars, and for star Crater J113615-105244 we also used the $445.4 \mathrm{~nm}$ line. We used the model atom described in Andrievsky et al. (2009).

The derived abundances for the two stars are given in Table 3 . The effects of systematic errors due to different assumptions on $T_{\text {eff }}, \log g$, and microturbulent velocities for star Crater J113613105227 are clearly visible in Table 4.

As a check of our method, we also analysed the X-Shooter spectrum of HD 165195 with MyGIsFOS. We assumed $T_{\text {eff }}=$ $4500 \mathrm{~K}, \log g=1.1$, and a microturbulence of $1.6 \mathrm{~km} \mathrm{~s}^{-1}$, as found by Bonifacio et al. (1999). The derived $[\mathrm{Fe} / \mathrm{H}]$ is -2.08 , very close to what was found by Bonifacio et al. $(1999,-1.92)$; this confirms the soundness of our method.

The derived abundances are consistent with the metallicity derived from the isochrone fits. The PARSEC ischrones assume solar-scaled abundances. Our detailed analysis reveals that the stars are instead $\alpha$-enhanced. Salaris et al. (1993) have shown that for metal-poor stars an $\alpha$-enhanced isochrone can be mimicked by a solar-scaled one of higher metallicity. Using their prescription and the mean of our measured $[\mathrm{Fe} / \mathrm{H}]$ and $[\alpha / \mathrm{Fe}]$, we find that our stars probably are compatible with an isochrone that is only about 0.1 dex more metal-rich than the one shown in Fig. 1. This is well within the errors of our abundance measurements and the isochrone-fitting procedure.

\section{Discussion}

Our spectroscopic results confirm the metallicity estimated from the photometry by Belokurov et al. (2014) and Laevens et al. (2014). The abundance pattern of the two stars does not show any signature that distinguishes it from halo stars. The $\alpha$ elements are enhanced over iron, and the iron peak elements follow iron. $\mathrm{Na}$ is underabundant by roughly a factor of two with respect to iron in both stars, as found in Galactic halo stars at this metallicity.

The radial velocity of the system is very interesting (see Sect. 2.2). Belokurov et al. (2014) have already pointed out that Crater, Leo IV, and Leo V all lie on a great circle with a pole at $(\alpha, \delta)=\left(83^{\circ} .1,-5^{\circ} .3\right)$. de Jong et al. (2010) suggested that Leo IV and Leo V are connected by a "bridge of stars" and could have been accreted by the Galaxy as a pair. Belokurov et al. (2014) suggested that Crater may in fact be a member of the same group of galaxies. Our measured radial velocity and confirmed distance from the DES photometry strongly supports this hypothesis. This is illustrated in Fig. 5, where we show the positional and kinematical properties of the three galaxies, together with the orbits computed by de Jong et al. (2010) under the assumption that Leo IV and Leo V have a common energy and angular momentum.

The association of Crater with the other two dwarf galaxies is evidence that favours the interpretation of Belokurov et al. (2014). It might be argued that Crater is a GC associated with either Leo IV or Leo V. This would make this whole system very exceptional because with the exception of the Magellanic Clouds, the only Milky Way satellites that harbour GCs are the two most massive dwarf spheroidals: Sagittarius and Fornax. The presence of a GC system seems to be associated with a high mass; it would be very unusual if Leo IV or Leo V, which have masses a factor of one thousand lower than Sagittarius or Fornax (McConnachie 2012), were indeed possessed of such satellites.

The fact that the Galactocentric radial velocity of Crater is so low implies that it is either close to peri-Galacton, or apoGalacticon, or on an orbit that is nearly circular. If the latter is true, or if the star is close to peri-Galacticon, then Crater is spending most of its time at large distances $(>150 \mathrm{kpc})$ from the Galactic centre, thus minimising the stripping effect of the Galactic tidal field on the gas. This could explain why this dwarf galaxy has been able to retain gas up to at least 2.2 Gyr ago and form a second generation of stars.

The radial velocities of the two stars observed by us differ by $10.2 \mathrm{~km} \mathrm{~s}^{-1}$. In Appendix A we show that, ignoring errors on the radial velocity, this implies a lower limit on the dispersion $\sigma_{v}>3.7 \mathrm{~km} \mathrm{~s}^{-1}$ with a $95 \%$ confidence.

Globular clusters are known to have several tight relations that connect their main structural parameters. One of them is the fundamental plane, which involves the central velocity dispersion, central density, and core radius (Djorgovski 1995). Another is a relation that involves the total luminosity and the velocity dispersion (Djorgovski \& Meylan 1994). We can therefore compare the velocity dispersion of Crater with those of GCs. For a total luminosity of -5.5 (Belokurov et al. 2014) or -4.3 (Laevens et al. 2014) for Crater, we expect a dispersion 
of $2.5 \div 4.0 \mathrm{~km} \mathrm{~s}^{-1}$. For a proper comparison, the ageing of the whole stellar population of Crater needs to be taken into account. Crater is about $5 \div 6$ Gyr younger than the average population of GCs, which is of the order of $12 \div 13$ Gyr. In the comparison, Crater should migrate at fainter magnitudes while not changing the velocity dispersion, which is barely affected. Although the comparison does not allow a strong disctinction between Crater being a GC or a dwarf galaxy, it certainly does not favour it being a GC.

Dwarf galaxies have dynamical masses higher than the masses estimated from their luminosities $\left(M_{\text {dyn }} \gg M_{*}\right)$, while for GCs the two masses are of the same order of magnitude. Our proposed lower limit on the velocity dispersion can be combined with the radius and distance of Crater to show that the dynamical mass of Crater is $M_{\text {Crater }}>1.5 M_{47 \text { Tuc }}$ (if we assume the radius measured by Laevens et al. 2014; $>1.8 M_{47 \text { Tuc }}$ if we use the larger radius measured by Belokurov et al. 2014, see Appendix for details). When coupled with the luminosities of Crater $\left(M_{V}=-4.3\right.$, Laevens et al. 2014) and 47 Tuc ( -9.42 , Harris 2010), this immediately tells us that Crater must contain a significant fraction of dark matter. This consideration could, by itself, answer the question and classify Crater as a dwarf galaxy. Only two stars have been measured, however, which weakens this argument very much (e.g. one of the two stars could be a binary or a radial velocity variable), and more measurements are certainly required to draw a firm conclusion.

Stars bluer than the turn-off may be explained as a younger population, which is another observation favouring the interpretation of Crater as a dwarf galaxy. Although it might be argued that these stars could be blue stragglers, they would appear to be too numerous for the cluster's luminosity. Momany (2015) showed that the logarithm of the ratio of the number of blue stragglers to horizontal branch stars in GCs has a clear anticorrelation with cluster luminosity. This anti-correlation is less tight for open clusters and dwarf galaxies that occupy different regions in this plane. If we count all stars to the blue of the turnoff in Crater as blue stragglers and compare to the number of HB stars (see the two boxes in Fig. 1), this would place it in a region of Fig. 6.4 of Momany (2015) that is mainly populated by dwarf galaxies and open clusters, but not by GCs. Therefore even interpreting the blue stars as blue stragglers would not support the notion that Crater is a typical GC.

The photometry suggests that the metallicity of this younger population has the same metallicity of the main population; this is expected. Such low-mass galaxies (Laevens et al. 2014 estimated $6.8 \times 10^{3} M_{\odot}$ ) are expected to show a low metallicity dispersion. The reason is that they cannot effectively retain supernova ejecta.

We summarise the four observations that all favour the interpretation of Crater as a dwarf galaxy.

1. The likely association of Crater with Leo IV and Leo V, on the basis of its radial velocity.

2. The population blueward of the turn-off in the colour-magnitude diagram, which can be interpreted as a population that is $4.5 \mathrm{Gyr}$ younger than the dominant population.

3. The lower limit to the velocity dispersion in the system implies that Crater is more massive than 47 Tuc. Since it is also less luminous than 47 Tuc, it must contain a significant fraction of dark matter.

\section{Crater does not lie on the fundamental plane of GCs.}

These observations are circumstantial, however, and cannot rule out the possibility that the system is a GC, if an atypical one. Nevertheless, from the information currently available, the dwarf galaxy hypothesis seems more plausible.

Acknowledgements. We are grateful to the anonymous referee, who helped us to improve the paper and pointed out to us that the almost horizontal track of the young population SGB does not follow the expected blue-straggler locus and that Crater must be near peri- or apo-galacticon or on a nearly circular orbit, providing an argument in favour of its ability to retain gas. The project was funded by FONDATION MERAC. P.B., E.C., P.F., M.S., F.S., and R.C. acknowledge support from the Programme National de Cosmologie et Galaxies (PNCG) and Programme National de Physique Stellaire (PNPS) of the Institut National de Sciences de l'Univers of CNRS. Support for L.S. was provided by Chile's Ministry of Economy, Development, and Tourism's Millennium Science Initiative through grant IC120009, awarded to The Millennium Institute of Astrophysics, MAS. S.Z. acknowledges that this research was supported in part by the National Science Foundation under Grant No. NSF PHY11-25915 and by PRIN INAF 2014 - CRA 1.05.01.94.05 "Star won't tell their ages to GAIA". S.Z. warmly thank P. Ochner for useful observations at the Asiago telescopes.

\section{References}

Abbott, T. G., Aldering, J. Annis, et al. 2005, ArXiv e-prints [arXiv: astro-ph/0510346]

Andrievsky, S. M., Spite, M., Korotin, S. A., et al. 2007, A\&A, 464, 1081 Andrievsky, S. M., Spite, M., Korotin, S. A., et al. 2009, A\&A, 494, 1083

Belokurov, V., Irwin, M. J., Koposov, S. E., et al. 2014, MNRAS, 441, 2124 Bevington, P. R., \& Robinson, D. K. 1992 (New York: McGraw-Hill) Bonifacio, P., Centurion, M., \& Molaro, P. 1999, MNRAS, 309, 533 Bressan, A., Marigo, P., Girardi, L., et al. 2012, MNRAS, 427, 127

Caffau, E., Bonifacio, P., François, P., et al. 2011, A\&A, 534, A4

Carlsson, M. 1986, Uppsala Astronomical Observatory Reports, 33

Castelli, F. 2005, MSAIS, 8, 25

Cayrel, R., Depagne, E., Spite, M., et al. 2004, A\&A, 416, 1117

Chambers, K. C., \& PS1 Science Consortium 2015, AAS, 225, \#332.01

Chen, Y.-P., Trager, S. C., Peletier, R. F., et al. 2014, A\&A, 565, A117

Dehnen, W., \& Binney, J. J. 1998, MNRAS, 298, 387

de Jong, J. T. A., Martin, N. F., Rix, H.-W., et al. 2010, ApJ, 710, 1664

Diehl, H. T., Abbott, T. M. C., Annis, J., et al. 2014, SPIE, 9149

Djorgovski, S. 1995, ApJ, 438, L29

Djorgovski, S., \& Meylan, G. 1994, AJ, 108, 1292

Goldoni, P. 2011, Astron. Nachr., 332, 227

Guinouard, I., Horville, D., Puech, M., et al. 2006, Proc. SPIE, 6273, 116

Harris, W. E. 2010, ArXiv e-prints [arXiv: 1012.3224]

Henden, A., \& Munari, U. 2014, Contributions of the Astronomical Observatory Skalnate Pleso, 43, 518

King, I. R. 1966, AJ, 71, 64

Korotin, S. A., \& Mishenina, T. V. 1999, Astron. Rep., 43, 533

Korotin, S. A., Andrievsky, S. M., \& Luck, R. E. 1999, A\&A, 351, 168

Kučinskas, A., Dobrovolskas, V., \& Bonifacio, P. 2014, A\&A, 568, L4

Kurucz, R. L. 2005, Mem. Soc. Astron. It. Supp., 8, 14

Laevens, B. P. M., Martin, N. F., Sesar, B., et al. 2014, ApJ, 786, L3

Mashonkina, L., Gehren, T., Shi, J.-R., Korn, A. J., Grupp, F., et al. 2011, A\&A, 528, A87

McConnachie, A. W. 2012, AJ, 144, 4

Momany, Y. 2015, A\&SS Lib., 413, 129

Monaco, L., Bellazzini, M., Bonifacio, P., et al. 2005, A\&A, 441, 141

Munari, U., Henden, A., Frigo, A., et al. 2014, AJ, 148, 81

Salaris, M., Chieffi, A., \& Straniero, O. 1993, ApJ, 414, 580

Sbordone, L., Caffau, E., Bonifacio, P., \& Duffau, S. 2014, A\&A, 564, A109

Schlegel, D. J., Finkbeiner, D. P., \& Davis, M. 1998, ApJ, 500, 525

Schönebeck, F., Puzia, T. H., Pasquali, A., et al. 2014, A\&A, 572, A13

Shanks, T., Belokurov, V., Chehade, B., et al. 2013, The Messenger, 154, 38

Spitzer, L. 1987, Dynamical evolution of globular clusters (Princeton: Princeton University Press), 191

Trager, S. C., Djorgovski, S., \& King, I. R. 1993, Structure and Dynamics of Globular Clusters, 50, 347

Vernet, J., Dekker, H., D’Odorico, S., et al. 2011, A\&A, 536, A105 
Table 3. Detailed abundances.

\begin{tabular}{|c|c|c|c|c|c|c|c|c|c|c|c|c|c|}
\hline Ion & $A(\mathrm{X}) \odot$ & $N$ & $A(\mathrm{X})$ & $\begin{array}{l}{[\mathrm{X} / \mathrm{H}]} \\
\mathrm{J} 11361\end{array}$ & $\begin{array}{c}\sigma \\
-10522\end{array}$ & {$[\mathrm{X} / \mathrm{Fe}]$} & $\sigma$ & $N$ & $A(\mathrm{X})$ & $\begin{array}{c}{[\mathrm{X} / \mathrm{H}]} \\
\mathrm{J} 113\end{array}$ & $\begin{array}{c}\sigma \\
15-105244\end{array}$ & {$[\mathrm{X} / \mathrm{Fe}]$} & $\sigma$ \\
\hline $\mathrm{Na} \mathrm{I}$ & 6.30 & 2 & 4.35 & -1.95 & 0.20 & -0.22 & 0.36 & 2 & 4.35 & -1.95 & 0.20 & -0.28 & 0.36 \\
\hline $\mathrm{Mg}_{\mathrm{I}}$ & 7.54 & 2 & 6.12 & -1.42 & 0.03 & 0.31 & 0.26 & 2 & 6.22 & -1.32 & 0.42 & 0.35 & 0.51 \\
\hline $\mathrm{CaI}$ & 6.33 & 4 & 4.96 & -1.37 & 0.23 & 0.36 & 0.35 & 4 & 5.10 & -1.23 & 0.11 & 0.44 & 0.30 \\
\hline Ti II & 4.90 & 3 & 3.36 & -1.54 & 0.19 & 0.19 & 0.20 & 2 & 3.21 & -1.69 & 0.20 & -0.02 & 0.34 \\
\hline V I & 4.00 & 1 & 2.11 & -1.89 & & -0.16 & & 1 & 2.23 & -1.77 & & -0.10 & \\
\hline Cr II & 5.64 & 2 & 3.82 & -1.82 & 0.03 & -0.09 & 0.26 & 1 & 4.40 & -1.24 & & 0.43 & \\
\hline Mn I & 5.37 & 2 & 3.74 & -1.63 & 0.09 & 0.10 & 0.28 & & & & & & \\
\hline $\mathrm{Fe} I$ & 7.52 & 58 & 5.79 & -1.73 & 0.26 & 0.00 & & 32 & 5.85 & -1.67 & 0.28 & 0.00 & \\
\hline $\mathrm{Fe}$ II & 7.52 & 2 & 5.67 & -1.85 & 0.05 & 0.00 & & 1 & 5.97 & -1.55 & & 0.00 & \\
\hline $\mathrm{Ni} I$ & 6.23 & 2 & 4.55 & -1.68 & 0.18 & 0.05 & 0.31 & 1 & 4.62 & -1.61 & & 0.05 & \\
\hline Ba II & 2.17 & 1 & 0.49 & -1.68 & & -0.01 & & 2 & 0.43 & -1.74 & 0.20 & -0.070 .34 & \\
\hline
\end{tabular}

Notes. For elements for which several lines are measured, the quoted error is the line-to-line scatter, except for Na I. For Crater J113613-105227 the two lines of the doublet where fitted together, and for Crater J113615-105244 only the D2 line was fitted. The quoted errors are estimated from the goodness-of-fit. For the elements for which only one line was measured, we provide no estimate of the error, but recommend to take the error estimate provided for Fe I, since for both stars this is the element with the largest number of measured lines, and the line-to-line scatter can be interpreted as mainly due to the noise in the spectrum.

Table 4. Variations in stellar parameters for different assumptions on $T_{\text {eff }}, \log g$, and microturbulent velocity.

\begin{tabular}{rcrrr}
\hline \hline $\begin{array}{r}T_{\text {eff }} \\
\mathrm{K}\end{array}$ & $\begin{array}{r}\log g \\
{[\mathrm{cgs}]}\end{array}$ & $\begin{array}{r}\xi \\
\mathrm{km} \mathrm{s}^{-1}\end{array}$ & $\begin{array}{r}{[\mathrm{Fe} / \mathrm{H}]} \\
\text { dex }\end{array}$ & $\begin{array}{r}{[\alpha / \mathrm{Fe}]} \\
\mathrm{dex}\end{array}$ \\
\hline \multicolumn{5}{c}{ J113613-105227 } \\
4475 & 1.24 & 2.0 & -1.89 & 0.34 \\
4575 & 1.24 & 2.0 & -1.73 & 0.34 \\
4675 & 1.24 & 2.0 & -1.60 & 0.36 \\
\hline \multicolumn{5}{c}{ Different assumed log $g$} \\
4575 & 1.04 & 2.0 & -1.73 & 0.39 \\
4575 & 1.24 & 2.0 & -1.73 & 0.34 \\
4575 & 1.44 & 2.0 & -1.74 & 0.30 \\
\hline \multicolumn{5}{c}{ Different assumed $\xi$} \\
4575 & 1.24 & 1.6 & -1.54 & 0.23 \\
4575 & 1.24 & 1.8 & -1.63 & 0.28 \\
4575 & 1.24 & 2.0 & -1.73 & 0.31 \\
4575 & 1.24 & 2.2 & -1.81 & 0.37 \\
4575 & 1.24 & 2.4 & -1.90 & 0.42 \\
\hline
\end{tabular}

$\log g$ from Fe ionisation equilibrium

\begin{tabular}{lllll}
4575 & 1.62 & 2.0 & -1.75 & 0.26 \\
\hline
\end{tabular}




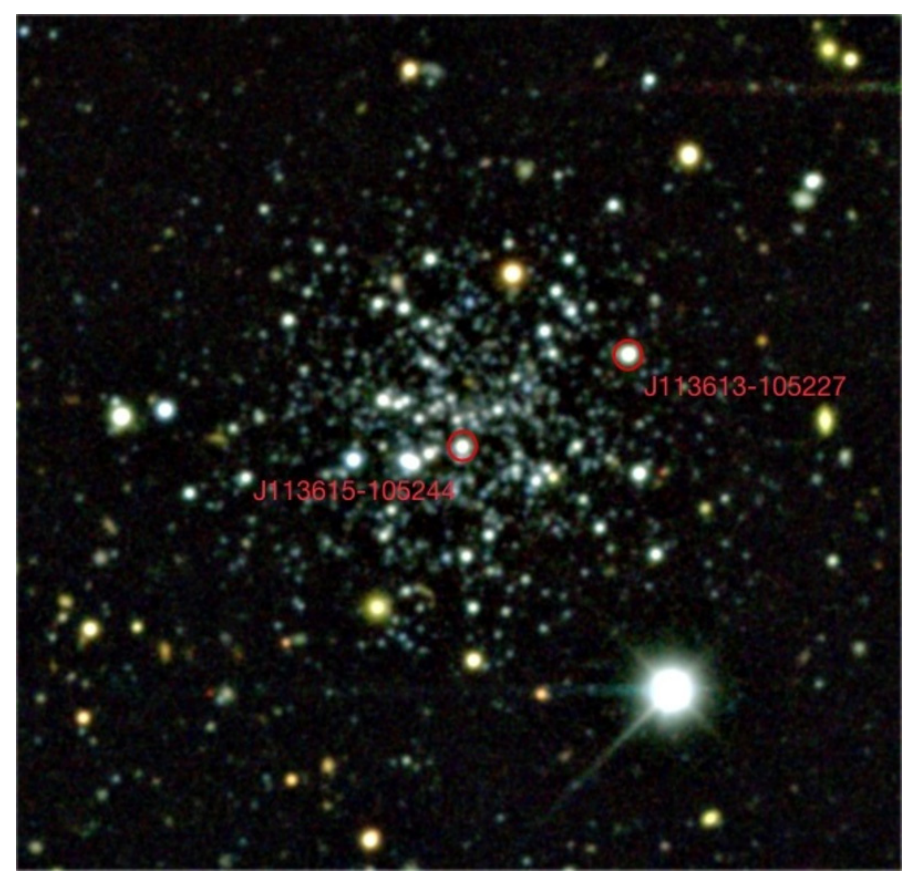

Fig. 4. Colour image created from DES, $g, r, i$ images showing the location of the two target stars.

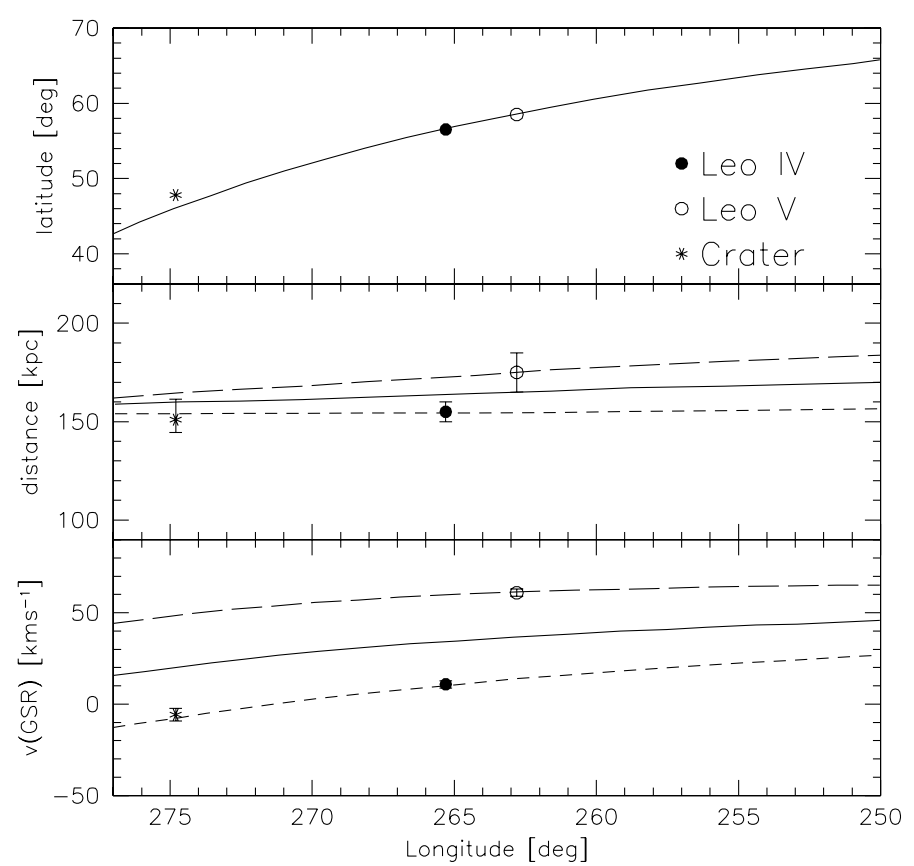

Fig. 5. Updated version of Fig. 7 of de Jong et al. (2010). The radial velocity (lower panel), distance (middle panel), and sky position (upper panel) of Crater (shown as a star) compared to those of Leo IV (empty dot) and Leo V (filled dot). The lines are the orbits for Leo IV and Leo V, computed by de Jong et al. (2010), assuming common energy and angular momentum for the two galaxies, using Leo IV and Leo V (dotted line) as initial conditions, or their mean properties (dashed line), respectively.

\section{Appendix A: Lower limit to the radial velocity dispersion}

The difference of two normal random variables with means $\left.\mu_{1}, \mu_{2}\right)$, and standard deviations $\sigma_{1}, \sigma_{2}$ follows a normal distribution, with $\sigma=\sqrt{\left(\sigma_{1}^{2}+\sigma_{2}^{2}\right)}$ and mean $\mu_{1}-\mu_{2}$. Assuming that the velocity distribution is normal and ignoring the measurement errors, the velocity difference between two stars is expected to be normally distributed with a mean of $0 \mathrm{~km} \mathrm{~s}^{-1}$ and a standard deviation of $\sqrt{2} \sigma_{v}$, where $\sigma_{v}$ is the standard deviation of the original distribution. Using the table on p. 253 of Bevington \& Robinson (1992), which provides the integral of a Gaussian distribution, we derive that the integral between $-1.96 \leq z \leq+1.96$ is 0.95 , where $z=\frac{|x-\mu|}{\sigma}$ and $\mu$ is the mean. In our case, $x=10.2 \mathrm{~km} \mathrm{~s}^{-1}, \mu=0 \mathrm{~km} \mathrm{~s}^{-1}$ and $\sigma=\sqrt{2} \sigma_{v}$; this implies that $z=1.96$ corresponds $\sigma_{v}=3.7 \mathrm{~km} \mathrm{~s}^{-1}$. In other words, there is a $95 \%$ probability that the parent velocity distribution has a $\sigma>3.7 \mathrm{~km} \mathrm{~s}^{-1}$. If we take into account the errors, no conclusion can be reached because the measured radial velocities of the two stars are also consistent with a zero radial velocity dispersion.

\section{Appendix B: Lower limit to the dynamical mass of Crater}

To derive a lower limit, we assumed that Crater and the globular cluster 47 Tuc are in dynamical equilibrium and that the virial theorem applies. For this system, the mass, $M$, can be expressed in terms of its velocity dispersion, $\sigma$, and exponential radius, $r_{\mathrm{h}}$ (see e.g. Spitzer 1987) :

$M=\frac{\sigma^{2} r_{\mathrm{h}}}{0.4 G}$

where $G$ is the gravitational constant. We thus may write

$\frac{M_{\text {Crater }}}{M_{47 \text { Tuc }}}=\frac{\sigma_{\text {Crater }}^{2}}{\sigma_{47 \text { Tuc }}^{2}} \frac{r_{\mathrm{hCrater}}}{r_{\mathrm{h} 47 \text { Tuc }}}$.

Because we only have a lower limit for $\sigma_{\text {Crater }}$, this becomes

$\frac{M_{\text {Crater }}}{M_{47 \text { Tuc }}}>\frac{\sigma_{\text {Crater }}^{2}}{\sigma_{47 \text { Tuc }}^{2}} \frac{r_{\text {hCrater }}}{r_{\mathrm{h} 47 \text { Tuc }}}$.

We assumed for Crater $\sigma_{\text {Crater }}>3.7 \mathrm{~km} \mathrm{~s}^{-1}, r_{\mathrm{hCrater}}=0.47^{\prime}$ (Laevens et al. 2014) or $r_{\mathrm{h} C r a t e r}=0.6^{\prime}$ (Belokurov et al. 2014) and a distance of $145 \mathrm{kpc}$ (Laevens et al. 2014). For 47 Tuc we assumed $\sigma_{47 \text { Tuc }}=7 \mathrm{~km} \mathrm{~s}^{-1}$ a median value from Kučinskas et al. (2014), $r_{\mathrm{h} 47 \text { Tuc }}=174^{\prime \prime}$ (Trager et al. 1993), and a distance of $4.5 \mathrm{kpc}$ (Harris 2010).

With these values, we obtain $\frac{M_{\text {Crater }}}{M_{47 \text { Tuc }}}>1.5$ for the radius measured by Laevens et al. (2014) and $\frac{M_{\text {Crater }}}{M_{47} \text { Tuc }} \gtrsim 1.9$ for the radius measured by Belokurov et al. (2014). Either way, it is clear that Crater is more massive (dynamically) than 47 Tuc. Even disregarding the velocity dispersions, it might have been suspected that this is the case because the radius of Crater is larger than that of 47 Tuc. Of course, if Crater is not in dynamical equlibrium, this reasoning does not hold. 